\title{
STM OBSERVATIONS OF FERROMAGNETIC CLUSTERS
}

\author{
A. WAWRO ${ }^{a, b}$ AND A. KASUY ${ }^{a}$ \\ ${ }^{a}$ Institute for Materials Research, Tohoku University \\ 2-1-1 Katahira, 980 Sendai, Japan \\ ${ }^{b}$ Institute of Physics, Polish Academy of Sciences \\ Al. Lotników 32/46, 02-668 Warsaw, Poland
}

\begin{abstract}
$\mathrm{Co}, \mathrm{Fe}$ and $\mathrm{Ni}$ clusters of nanometer size, deposited on silicon and graphite (highly oriented pyrolytic graphite), were observed by a scanning tunneling microscope. Deposition as well as the scanning tunneling microscope measurements were carried out in an ultrahigh vacuum system at room temperature. Detailed analysis of Co cluster height was done with the scanning tunneling microscope equipped with a ferromagnetic tip in the magnetic field up to $70 \mathrm{Oe}$. It is found that bigger clusters (few nanometers in height) exhibit a dependence of their apparent height on applied magnetic field. We propose that such behaviour originates from the ferromagnetic ordering of cluster and associate this effect to spin polarized tunneling.
\end{abstract}

PACS numbers: $34.80 . \mathrm{Nz}, 36.40 . \mathrm{Cg}, 61.16 . \mathrm{Ch}$

\section{Introduction}

Clusters - agglomerations of atoms, which cover the range from dimers to particles $100 \mathrm{~nm}$ in diameter - have been of great interest since they provide unique systems to understand the transition from atomic or molecular level to bulk condensed matter. Because of small size and high fraction of atoms located on the surface, their properties differ substantially from the bulk material. Recently, an attention has been focused on the magnetic properties of clusters [1-3].

In this paper we study the possibility of deposition of ferromagnetic clusters, few nanometer in size, on silicon and graphite substrates. For this purpose we use the scanning tunneling microscope (STM), which is an unique device to study such small and nonregular objects on the surface. In the past we successfully formed a regular array of gold clusters $3 \mathrm{~nm}$ in diameter and located $10 \mathrm{~nm}$ apart each other [4]. Similar array of the ferromagnetic clusters is promising for ultrahigh density magnetic memory carrier. Our preliminary results reveal that detection of magnetization reversal in such small particles is possible with the use of STM equipped with a ferromagnetic tip. 


\section{Experimental}

Whole experiment, i.e. cluster deposition and STM observations, were performed in an ultrahigh vacuum system in $2 \times 10^{-10} \mathrm{Tr}$ at room temperature. Highly oriented pyrolytic graphite (HOPG) cleaved in air and chemically etched silicon wafers were used as substrates. They were heated up to $600^{\circ} \mathrm{C}$ and $1230^{\circ} \mathrm{C}$, respectively, after transferring to the vacuum chamber. Such treatment resulted in free from contamination, well reconstructed substrate surfaces. We used two different techniques to produce $\mathrm{Co}, \mathrm{Fe}$ and $\mathrm{Ni}$ clusters: laser ablation from appropriate target illuminated by a $\mathrm{KrF}$ excimer laser and field assisted deposition from the STM tip. In this latter method the STM tip was approached to the surface, followed by applying a sequence of bias pulses and finally carefully withdrawn from the surface. Such procedure made possible to deposit clusters in a controlled way. The same tip was used for STM measurements after field assisted deposition. The magnetic field (up to $70 \mathrm{Oe}$ ) was generated by a coil placed on the STM head. The ferromagnetic tips were etched chemically in standard way in $\mathrm{HCl}$ acid. They were magnetized in the field of $3.5 \mathrm{kOe}$ parallel to their axis before the experiment. In order to clean the tip the scanning in UHV with high tunneling current and numerous predepositions were performed.

\section{Results and discussion}

The analyzed clusters were produced by two different methods: on graphite by laser ablation of appropriate target and on silicon by field assisted deposition from the STM tip. It is found that the size of clusters depends on deposition technique. All clusters deposited by laser ablation are smaller -1 to $3 \mathrm{~nm}$ in diameter and less than $1 \mathrm{~nm}$ in height. Rough estimations provide that they consist of several tens to hundreds of atoms. The size of particles produced by bias pulse application between the ferromagnetic tip and the substrate depends on pulse height, its duration and the distance between the STM tip and the substrate. Therefore their size is easily controlled by these parameters. In the preliminary stage of experiment we fabricated reproducibly clusters several tens times larger than those deposited by laser ablation from the targets: 6 to $10 \mathrm{~nm}$ in diameter and 2 to $4 \mathrm{~nm}$ in height. Most of them were located close to the step edges of the substrate. The width of terraces in our substrates was relatively narrow $c a .60 \mathrm{~nm}$. Because the lines of electric field are distributed more densely near the sharp points of the conducting surface and electric field is higher there, this explains why clusters are located just in the environment of steps.

We chose Co clusters to study by the STM in the magnetic field applied in perpendicular direction to the sample surface. The topography images recorded without magnetic field and in maximum magnetic field available in our system, i.e. $70 \mathrm{Oe}$, were compared. The tunneling parameters like intensity of tunneling current, bias and feedback loop parameters remained unchanged during switching the magnetic field. Consecutive scans, being a subject of further analysis, were performed under the same conditions for better statistic. It occasionally happened that the scanning tip hit the deposited cluster. Such results were excluded from analysis to be sure that the shape and size of clusters remained unperturbed during the experiment. 

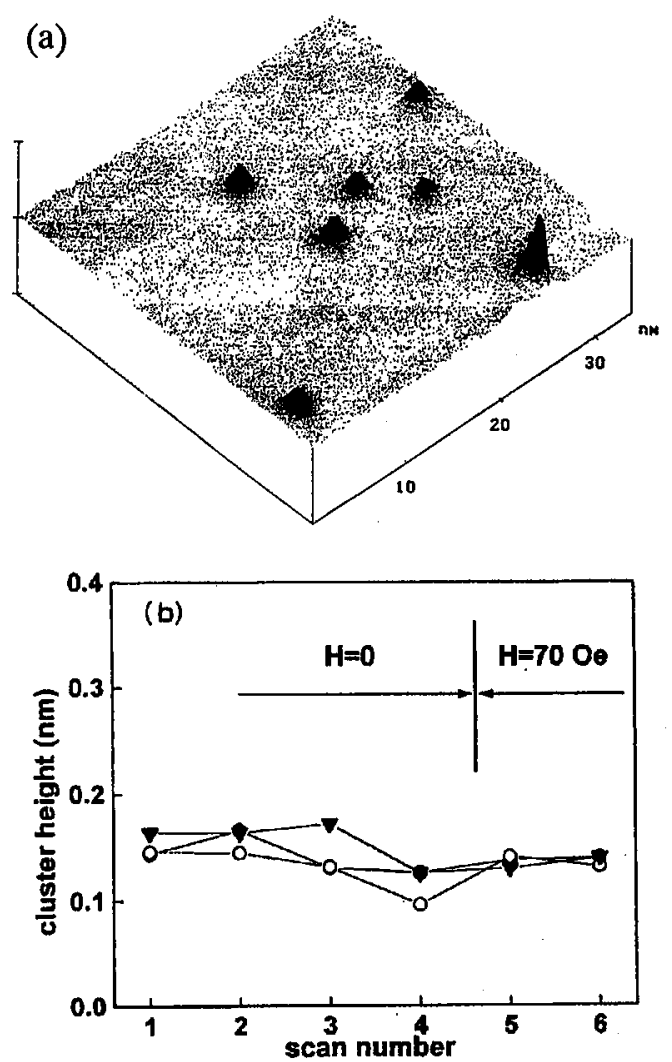

Fig. 1. (a) The group of Co clusters deposited by laser ablation on HOPG substrate. Their height is comparable to one monolayer. (b) Height dependence of some clusters shown in (a) taken from consecutive scans without magnetic field (scan numbers 1-4) and in 70 Oe (scan numbers 5,6 ).

The STM images of deposited Co clusters and their height dependence on the magnetic field is shown in Figs. 1 and 2. The height of objects, measured by the STM relatively to the substrate surface level, is most reliable, because it does not depend on the STM tip shape, contrary to the lateral distances. It was determined as an average value obtained from cross-sections analyses taken in different directions. The height of small clusters (Fig. 1b) does not change with the magnetic field. First four points (scan numbers 1- 4 ) for each cluster were obtained in absence of the magnetic field. Two last values were measured in the magnetic field of $70 \mathrm{Oe}$ (scan numbers 5,6 ). No difference in height is noticed: the measured heights are around $0.15 \mathrm{~nm}$, which corresponds to one monolayer, both in and without magnetic field. The height of bigger clusters changes with magnetic field (Fig. 2b). Its value is $c a .3 .47 \mathrm{~nm}$ in as deposited state without magnetic field (scan numbers 1-3) and increases by about $0.08 \mathrm{~nm}$ (scan numbers 4 and 5) in magnetic 

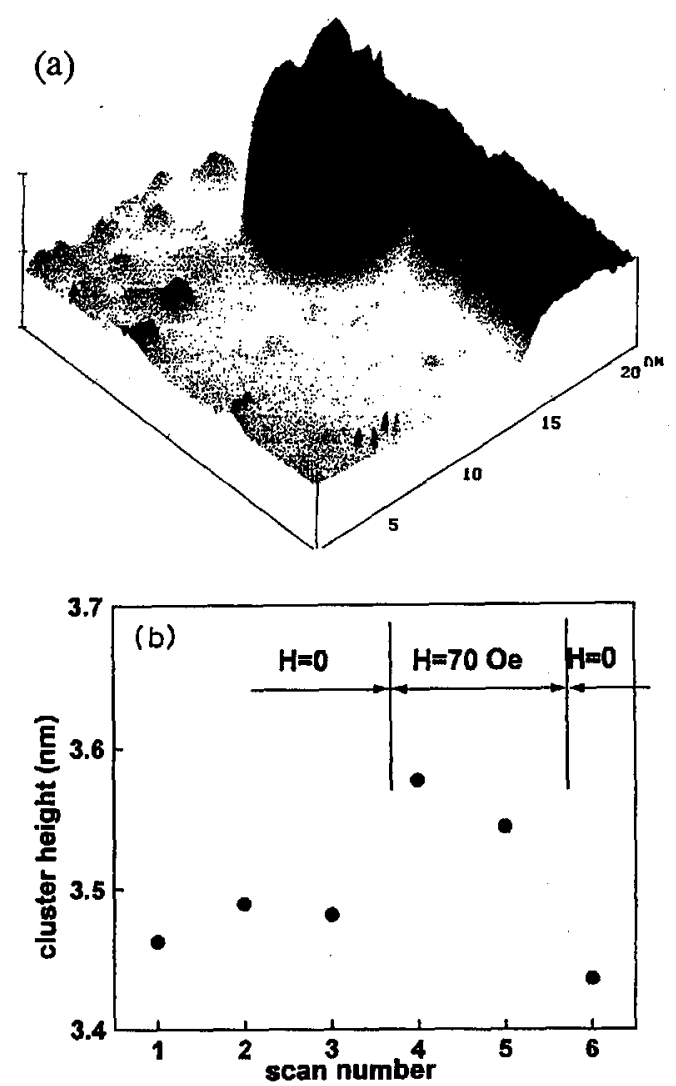

Fig. 2. (a) Co cluster deposited from the STM tip located close to the terrace edge. (b) Its height measured without magnetic field (scan numbers 1-3), in 70 Oe (scan numbers 4,5 ) and again without magnetic field (scan number 6).

field of 70 Oe. Again, the apparent height of cluster decreases substantially (scan number 6 ), when the magnetic field is switched off. It is worth mentioning that the height of nonmagnetic objects like steps of the silicon substrate remains unchanged within experimental accuracy.

In experiments on free clusters the ferromagnetic ordering has been found even for the smallest particles [3], although the superparamagnetic behaviour i.e. spatial rotation of the magnetic moment may occur at higher temperatures. The magnetic moment per atom is atom-like in clusters consisting of 30 atoms and decreases with the size increase to the bulk value. This limit is reached for Co particles formed by 450 atoms. It is expected that particles smaller than several tens of nanometer in diameter should have single domain magnetic structure [5]. Recently it has been found that Co dots which have a diameter of $200-250 \mathrm{~nm}$ exhibit also single domain structure [6]. When the STM tip is magnetic the imaging 
of the sample depends on its magnetization. Theoretical considerations [7] reveal that the intensity of tunneling current depends on mutual orientation of the magnetization vectors in ferromagnetic electrodes. This prediction was confirmed in the experiments performed on magnetic samples $[8,9]$.

It is reasonable to expect that our clusters have single domain structure. The orientation of magnetic vector in cluster and in the apex of the tip without magnetic field is determined by crystalline and/or shape anisotropy and therefore are, most probably, not parallel. When the magnetic field is switched on, these vectors rotate towards parallel alignment. As a consequence, the tunneling current increases and microscope, which operates in constant current mode, records the bigger height of cluster. While the magnetic field decreases, the apparent cluster height decreases due to divergence of magnetic vectors. Although the exact directions of magnetization vectors in these two objects remain a puzzle, the mutual rotation due to magnetic field is clearly detected. Because the parameters of tunneling process remain the same during consecutive scans we believe that changes of measured cluster height with switching magnetic field originate from ferromagnetic structure and this effect is explained in terms of spin polarized electrons. We associate the lack of similar effect in small particles (Fig. 1) to their superparamagnetic character. The magnetic field of $70 \mathrm{Oe}$ is probably too weak to anchor the magnetization vector in one direction.

\section{Summary}

We studied the possibilities to produce ferromagnetic clusters on various substrates. The STM investigations show that wide range of cluster sizes is available in two applied deposition techniques. Clusters obtained by laser ablation have diameter of $2-3 \mathrm{~nm}$ and height less than $1 \mathrm{~nm}$, whereas those produced by field associated deposition from ferromagnetic STM tip are several tens times larger. The STM measurements show that apparent height of bigger Co clusters changes under the influence of magnetic field. This is a consequence of spin polarized tunneling current changes due to magnetization reversal of cluster and apex of the tip. The lack of similar effect in smaller clusters is associated to their superparamagnetic state even in magnetic field of 70 Oe.

\section{References}

[1] D.M. Cox, D.J. Trevor, R.L. Whetten, E.A. Rohlfing, A. Kaldor, Phys. Rev. B 32, 7290 (1985).

[2] J.P. Bucher, D.C. Douglass, L.A. Bloomfield, Phys. Rev. Lett. 66, 3052 (1991).

[3] I.M.L. Billas, A. Châtelain, W.A. de Heer, Science 265, 1682 (1994).

[4] A. Wawro, A. Kasuya, R. Czajka, N. Horiguchi, Y. Nishina, Surf. Coat. Technol. 67, 173 (1994).

[5] A.H. Morrish, Fizyczne podstawy magnetyzmu, PWN, Warszawa 1970, p. 321.

[6] M. Löhndorf, A. Wadas, G. Lütjering, D. Weiss, R. Wiesendanger, Z. Phys. B 101, 1 (1996).

[7] J. Slonczewski, Phys. Rev. B 39, 6995 (1989). 
[8] R. Wiesendanger, H.-J. Güntherodt, G. Güntherodt, R.J. Gambino, R. Ruf, Phys. Rev. Lett. 65, 247 (1990).

[9] R. Wiesendanger, I.V. Shvets, D. Bürgeler, G. Tarrach, H.-J. Güntherodt, J.M.D. Coey, Z. Phys. B 86, 1 (1992). 